\title{
Comparison of Ureteroscopic Pneumatic Lithotripsy and Extracorporeal Shock Wave Lithotripsy for Proximal Ureteral Calculi
}

Muhammad Fazal Ur Rehman ${ }^{1}$, Muhammad Adnan ${ }^{1}$, Ali Hassan III ${ }^{2}$, Fawad Humayun Akhtar ${ }^{3}$, Naseem Javed ${ }^{4}$, Farman Ali $^{5}$

1. Urology, Bakhtawar Amin Medical and Dental College, Multan, PAK 2. Urology, University of Lahore Teaching Hospital, Lahore, PAK 3. Urology, Children Hospital and Institute of Child Health, Lahore, PAK 4. Paediatric Urology, Children Hospital and Institute of Child Health, Lahore, PAK 5. Internal Medicine, District Headquarter Hospital, Khanewal, PAK

Corresponding author: Farman Ali, chfarmanali358@gmail.com

\section{Abstract}

\section{Objective}

The goal of this study was to compare the effectiveness and complications of ureteroscopic pneumatic lithotripsy (URS) and extracorporeal shock wave lithotripsy (SWL) in the management of patients with proximal ureteral stones.

\section{Methods}

In this trial, 150 patients presenting with proximal ureteral stones at the Department of Urology of Nishter Hospital Multan from November 2018 to November 2019 were allocated 1:1 to undergo URS or SWL. The presence of stone fragments $<4 \mathrm{~mm}$ on follow-up was regarded as being stone free. The study outcomes included stone-free rates after first, second, and third treatment sessions and stone retropulsion into the kidneys.

\section{Results}

A total of 75 patients each underwent URS and SWL. The mean procedure times for SWL and URS were $61.61 \pm 3.21$ and $85.01 \pm 6.75$ minutes, respectively $(\mathrm{P}=0.000)$, and the mean numbers of procedures were $1.51 \pm 0.49$ and $1.01 \pm 0.42$, respectively $(\mathrm{P}=0.000)$. Stone-free rates after the first, second, and third sessions of SWL were $64 \%, 77.3 \%$, and $94.7 \%$, respectively, whereas stone-free rates after the first, second, and third sessions of URS were $86.7 \%, 92 \%$, and $100 \%$, respectively. Rates of stone retropulsion into the kidneys in the SWL and URS groups were $0 \%$ and $6.7 \%$, respectively $(\mathrm{P}=0.000)$.

\section{Conclusion}

Received 03/30/2020

Review began 04/17/2020 Review ended 04/17/2020 Published 04/26/2020

\section{(c) Copyright 2020}

Ur Rehman et al. This is an open access article distributed under the terms of the Creative Commons Attribution License CC-BY 4.0., which permits unrestricted use, distribution, and reproduction in any medium, provided the original author and source are credited.
Compared with SWL, URS had significantly higher stone-free rates in patients with proximal ureteral stones. Treatment costs and hospital stay were lower in the SWL group, whereas complication rates were comparable.

Categories: Urology

Keywords: complications, eswl, stone-free rate, proximal ureter stone, urs

\section{Introduction}

Urolithiasis is the most common worldwide cause of morbidity in patients with diseases of the urinary tract [1]. Minimally invasive procedures have eased the removal of urinary tract stones [2]. Although ureteral stones were previously managed by open ureter lithotomy, newer techniques, including shock wave lithotripsy (SWL), refinement of semirigid ureter scopes, flexible ureterorenoscopy, and certain laparoscopic procedures have been shown safe and effective in the treatment of ureteral stones, in adults and children [35].

SWL is a minimally invasive technique that can be performed on an outpatient basis for the treatment of proximal ureteral calculi [6]. SWL has adverse effects, including long treatment time, a high percentage of patients requiring retreatment, and lack of patient compliance [7].

Ureteroscopic pneumatic lithotripsy (URS) treatment is another technique increasingly used to remove ureteral calculi, especially distal calculi [8]. The success rate of semirigid URS was shown to be higher for proximal than for distal stones [9]. Because URS is associated with a higher success rate after a single session and a lower rate of multiple visits, URS is preferred to SWL in areas in which healthcare facilities are limited 
[10]. The present study compared the outcomes of URS and SWL in the management of proximal ureteral calculi.

\section{Materials And Methods}

In this trial, 150 patients presenting with proximal ureteral stones at the Department of Urology of Nishter Hospital Multan from November 2018 to November 2019 were allocated 1:1 to undergo URS or SWL. After patients were counseled and informed of the advantages and disadvantages of both procedures, patients were allowed to undergo SWL or URS. All patients provided written informed consent.

Patients were diagnosed clinically, based on history, physical examination and X-rays, ultrasound, and CT of the kidneys, ureter, and bladder (KUB). Patients aged $>18$ years with proximal radiopaque calculi $<2 \mathrm{~cm}$ in diameter were enrolled. Patients with urinary tract infection, a previous history of ureteral stone surgery, or a coagulation disorder were excluded, as were pregnant women.

Before URS, patients were administered prophylactic antibodies by intravenous injection. URS was performed under general anesthesia with a video monitor attached to the cystoscope. After a guidewire was inserted into the ureter, a rigid ureteroscope of 9F/11F was used. Stones were broken with a Swiss pneumatic LithoClast, and stone fragments removed with a Dormia basket. Intravenous administration of prophylactic antibodies was continued until 24 hours after the procedure, following which the patients were switched to oral antibiotics for one week. Folly's catheter was removed on the first postoperative day. Patients were followed up for three months by X-ray or ultrasound of the KUP. The procedure was considered successful if remnant stone fragments were $<4 \mathrm{~mm}$ in size.

SWL was performed using a Storz Modulith Electromagnetic lithotripter (Karl Storz Lithotripsy-America Inc, Atlanta, GA). Stones were identified by ultrasound and fluoroscopy. Patients were administered 90 shock waves at energy level 2 for one minute, followed by 200 shocks at level 3 or 4 . Patients who experienced pain were administered intravenous injections of nalbuphine. Patients with large residual stones were advised to return for a second or even a third session. Patients were monitored via follow-up for three months by X-ray or ultrasound of the KUP. The procedure was considered successful if remnant stone fragments were smaller than $4 \mathrm{~mm}$.

\section{Results}

Of the 150 patients enrolled in this study, 75 underwent SWL and 75 underwent URS. Gender distribution, mean age, body mass index (BMI), stone size, skin to stone distance, and Hounsfield units (HU) did not differ significantly in the two groups (Table 1). Of the 75 patients in the SWL group, 32 (42.7\%) had right-sided and 43 (57.3\%) had left-sided stones, comparable to the 31 (41.3\%) patients with right-sided stones and 44 (58.7\%) with left-sided stones in the URS group. The prevalence of hypertension was significantly lower in the SWL than in the URS group ( $28.0 \%$ vs. $45.3 \%, \mathrm{P}=0.028)$, whereas the prevalence of diabetes mellitus was similar (41.3\% vs. $37.3 \%$, $\mathrm{P}=0.616)$. 


\section{Cureus}

\begin{tabular}{|c|c|c|c|}
\hline Characteristics & SWL group $(n=75)$ & URS group ( $n=75$ ) & P-value \\
\hline Age (years) & $41.21 \pm 3.15$ & $40.98 \pm 3.73$ & 0.689 \\
\hline BMI (kg/m²) & $23.98 \pm 1.87$ & $10.51 \pm 1.82$ & 0.484 \\
\hline Skin to stone distance & $9.33 \pm 1.54$ & $9.34 \pm 1.52$ & 0.957 \\
\hline Stone size $(\mathrm{cm})$ & $10.51 \pm 2.31$ & $24.19 \pm 1.92$ & 0.969 \\
\hline Hounsfield units of stones & $799.77 \pm 14.47$ & $801.17 \pm 11.8$ & 0.519 \\
\hline \multicolumn{4}{|l|}{ Gender } \\
\hline Male & $45(60 \%)$ & $47(62.7 \%)$ & \multirow{2}{*}{0.737} \\
\hline Female & $30(40 \%)$ & $28(37.3 \%)$ & \\
\hline \multicolumn{4}{|l|}{ Side } \\
\hline Right side & $32(42.7 \%)$ & $31(41.3 \%)$ & \multirow{2}{*}{0.869} \\
\hline Left side & $43(57.3 \%)$ & $44(58.7 \%)$ & \\
\hline Hypertension & $21(28 \%)$ & 34 (45.3\%) & 0.028 \\
\hline Diabetes mellitus & $31(41.3 \%)$ & $28(37.3 \%)$ & 0.616 \\
\hline
\end{tabular}

TABLE 1: Demographic characteristics of patients with proximal ureteral stones treated with shock wave lithotripsy and ureteroscopic pneumatic lithotripsy

Results reported as mean \pm standard deviation or as number (\%).

BMI, body mass index; SWL, shock wave lithotripsy; URS ureteroscopic pneumatic lithotripsy.

The mean procedure time was significantly shorter ( $61.61 \pm 3.21$ vs. $85.01 \pm 6.75$ minutes, $\mathrm{P}=0.000$ ), while the mean number of sessions was significantly higher $(1.51 \pm 0.49$ vs. $1.01 \pm 0.42, \mathrm{P}=0.000)$ in the SWL group than in the URS group. Stone-free rates after the first, second, and third sessions were $64 \%, 77.3 \%$, and $94.7 \%$, respectively, in the SWL group, and $86.7 \%, 02 \%$, and $100 \%$, respectively, in the URS group (Table 2).

\begin{tabular}{|c|c|c|c|}
\hline Characteristics & SWL group ( $n=75$ ) & URS group $(n=75)$ & P-value \\
\hline Procedural time (minutes) & $61.61 \pm 3.21$ & $85.01 \pm 6.75$ & 0.000 \\
\hline Number of sessions (procedures) & $1.51 \pm 0.49$ & $1.01 \pm 0.42$ & 0.000 \\
\hline Hospital stay (days) & -- & $1.38 \pm 0.21$ & -- \\
\hline Stone-free rate after first session & $48(64 \%)$ & $65(86.7 \%)$ & 0.001 \\
\hline Stone-free rate after second session & $58(77.3 \%)$ & $69(92 \%)$ & 0.013 \\
\hline Stone-free rate after third session & $71(94.7 \%)$ & $75(100 \%)$ & 0.000 \\
\hline Stone retropulsıon Into kıdney (URS) & $0(0 \%)$ & $5(6.1 \%)$ & 0.000 \\
\hline
\end{tabular}

TABLE 2: Procedural outcomes of patients with proximal ureteral stones treated with shock wave lithotripsy and ureteroscopic pneumatic lithotripsy

Results reported as mean \pm standard deviation or as number (\%).

SWL, shock wave lithotripsy; URS ureteroscopic pneumatic lithotripsy. 


\section{Cureus}

two groups (Table 3).

\begin{tabular}{|c|c|c|c|}
\hline Clavien grade & SWL group $(n=75)$ & URS goup ( $n=75)$ & P-value \\
\hline 0=no complications & $59(78.7 \%)$ & $60(80 \%)$ & 0.840 \\
\hline 1=deviation from normal post procedural course without need for intervention & $7(9.3 \%)$ & $9(12 \%)$ & 0.597 \\
\hline $2=$ mild complications needing intervention & $1(1.3 \%)$ & $1(1.3 \%)$ & 1.000 \\
\hline $3 a=$ postprocedural complications needing intervention without use of general anesthesia & $6(8 \%)$ & $5(6.7 \%)$ & 0.754 \\
\hline $3 \mathrm{~b}=$ complications needing intervention under general anesthesia & $3(4 \%)$ & $8(10.7 \%)$ & 0.117 \\
\hline $4 a=$ life-threatening complication needing intensive care management & $4(5.3 \%)$ & $1(1.3 \%)$ & 0.172 \\
\hline $4 b=$ life-threatening complication needing intensive care management & $4(5.3 \%)$ & $1(1.3 \%)$ & 0.172 \\
\hline $5=$ death & $2(2.7 \%)$ & $1(1.3 \%)$ & 0.560 \\
\hline \multicolumn{4}{|c|}{$\begin{array}{l}\text { TABLE 3: Clavien grading system scores in patients with proximal ureteral stones treated with } \\
\text { shock wave lithotripsy and ureteroscopic pneumatic lithotripsy }\end{array}$} \\
\hline \multicolumn{4}{|l|}{ Results reported as number (\%). } \\
\hline SWL, shock wave lithotripsy; URS ureteroscopic pneumatic lithotripsy. & & & \\
\hline
\end{tabular}

\section{Discussion}

Management of patients with urinary lithiasis includes minimally invasive procedures as well as noninvasive modalities such as SWL, thereby reducing the need for open surgery [11]. SWL has been reported to be optimal for proximal stones of the ureter, with antracarporeal lithotripsy being safe for these patients [12]. URS has also been reported safe, with a success rate of $80 \%$ in the removal of proximal ureteral stones and with a higher success rate in patients with smaller than larger stones [13]. Experience and skill are necessary to perform URS [14]. Although both SWL and URS are effective and safe, SWL was found to be more effective.

Aboutaleb et al. concluded in his study that although SWL was shown to be safe for the removal ureteral stones, with a higher stone-free rate than URS, the complication rate is higher and hospital stay longer in patients who underwent SWL [15]. A comparison of URS and SWL in patients with proximal stones found no significant differences between these two modalities.

The success rates of both procedures are reduced in patients with a BMI $>30 \mathrm{~kg} / \mathrm{m}^{2}$ and stones with $>500 \mathrm{HU}$ [16]. A second study reported similar results that both techniques are equally effective for stone removal, especially for proximal ureteral stones [17].

A comparison of complications in patients undergoing stone removal reported that all complications in patients who underwent SWL were minor nature, with the most common being loin pain, occurring in $21 \%$ of patients [18]. A study comparing URS and SWL for ureteral calculi reported that both had similar complication rates of about $4 \%$, whereas episodic treatment was not possible [19].

\section{Conclusions}

URS had significantly higher stone-free rates in patients with proximal ureteral stones as compared with SWL. Treatment cost and hospital stay were lower in the SWL group, whereas complication rates were comparable.

\section{Additional Information \\ Disclosures}

Human subjects: Consent was obtained by all participants in this study. Bakhtawar Amin Medical and Dental collage, Multan issued approval 32/URO/BMDC-2018. Animal subjects: All authors have confirmed that this study did not involve animal subjects or tissue. Conflicts of interest: In compliance with the ICMJE uniform disclosure form, all authors declare the following: Payment/services info: All authors have declared that no financial support was received from any organization for the submitted work. Financial relationships: All authors have declared that they have no financial relationships at present or within the 
previous three years with any organizations that might have an interest in the submitted work. Other relationships: All authors have declared that there are no other relationships or activities that could appear to have influenced the submitted work.

\section{References}

1. Cui X, Ji F, Yan H, et al.: Comparison between extracorporeal shock wave lithotripsy and ureteroscopic lithotripsy for treating large proximal ureteral stones: a meta-analysis. Urology. 2015, 85:748-756. 10.1016/j.urology.2014.11.041

2. Kumar A, Nanda B, Kumar N, Kumar R, Vasudeva P, Mohanty NK: A prospective randomized comparison between shockwave lithotripsy and semirigid ureteroscopy for upper ureteral stones $<2 \mathrm{~cm}$ : a single center experience. J Endourol. 2015, 29:47-51. 10.1089/end.2012.0493

3. Hyams ES, Monga M, Pearle MS, et al.: A prospective, multi-institutional study of flexible ureteroscopy for proximal ureteral stones smaller than 2 cm. J Urol. 2015, 193:165-169. 10.1016/j.juro.2014.07.002

4. Cone EB, Pareek G, Ursiny M, Eisner B: Cost-effectiveness comparison of ureteral calculi treated with ureteroscopic laser lithotripsy versus shockwave lithotripsy. World J Urol. 2017, 35:161-166. 10.1007/s00345-016-1842-2

5. Dean LA, Adashi EY: Repealed and replaced: SGR gives way to value-based Medicare payment reform . Am J Med. 2015, 128:1052-1053. 10.1016/j.amjmed.2015.04.027

6. Rana AJ, Bozic KJ: Bundled payments in orthopaedics. Clin Orthop Relat Res. 2015, 473:422-425. 10.1007/s11999-014-3520-2

7. Tran TY, McGillen K, Cone EB, Pareek G: Triple D Score is a reportable predictor of shockwave lithotripsy stone-free rates. J Endourol. 2015, 29:226-230. 10.1089/end.2014.0212

8. Drake T, Grivas N, Dabestani S, et al.: What are the benefits and harms of ureteroscopy compared with shock-wave lithotripsy in the treatment of upper ureteral stones? A systematic review. Eur Urol. 2017, 72:772-786. 10.1016/j.eururo.2017.04.016

9. Niwa N, Matsumoto K, Miyahara M, et al.: Simple and practical nomograms for predicting the stone-free rate after shock wave lithotripsy in patients with a solitary upper ureteral stone. World J Urol. 2017, 35:1455-1461. 10.1007/s00345-017-2014-8

10. Ichiyanagi O, Nagaoka A, Izumi T, Kawamura Y, Kato T: Age-related delay in urinary stone clearance in elderly patients with solitary proximal ureteral calculi treated by extracorporeal shock wave lithotripsy. Urolithiasis. 2015, 43:419-426. 10.1007/s00240-015-0783-3

11. Nasseh H, Pourreza F, Kazemnejad Leyli E, Zohari Nobijari T, Baghani Aval H: Laparoscopic transperitoneal ureterolithotomy: a single-center experience. J Laparoendosc Adv Surg Tech A. 2013, 23:495-499. 10.1089/lap.2012.0187

12. Manzoor S, Hashmi AH, Sohail MA, Mahar F, Bhatti S, Khuhro AQ: Extracorporeal shock wave lithotripsy (ESWL) vs. ureterorenoscopic (URS) manipulation in proximal ureteral stone. J Coll Physicians Surg Pak. 2013, 23:726-730.

13. Islam M, Malik A: Ureteroscopic pneumatic versus extracorporeal shock wave lithotripsy for lower ureteral stones. J Coll Physicians Surg Pak. 2012, 22:444-447. 07.2012/JCPSP.444447

14. Librenjak D, Šitum M, Gugić D, Milostić K, Duvnjak M: Ureterorenoscopic treatment of ureteral stones-influence of operator's experience and skill on the procedure outcome. Croat Med J. 2011, 52:55-60. $10.3325 / \mathrm{cmj} .2011 .52 .55$

15. Aboutaleb H, Omar M, Salem S, Elshazly M: Management of upper ureteral stones exceeding $15 \mathrm{~mm}$ in diameter: shock wave lithotripsy versus semirigid ureteroscopy with holmium:yttrium-aluminum-garnet laser lithotripsy. SAGE Open Med. 2016, 4:2050312116685180. 10.1177/2050312116685180

16. Massoud AM, Abdelbary AM, Al-Dessoukey AA, Moussa AS, Zayed AS, Mahmmoud O: The success of extracorporeal shock-wave lithotripsy based on the stone-attenuation value from non-contrast computed tomography. Arab J Urol. 2014, 12:155-161. 10.1016/j.aju.2014.01.002

17. Alameddine M, Azab MM, Nassir AA: Semirigid ureteroscopy: Proximal versus distal ureteral stones. Urol Ann. 2016, 8:84-86. 10.4103/0974-7796.171495

18. Al-Marhoon MS, Shareef O, Al-Habsi IS, Mathew J, Venkiteswaran KP: Extracorporeal shock-wave lithotripsy success rate and complications: initial experience at Sultan Qaboos University Hospital. Oman Med J. 2013, 28:255-259. 10.5001/omj.2013.72

19. Salem HK: A prospective randomized study comparing shock wave lithotripsy and semirigid ureteroscopy for the management of proximal ureteral calculi. Urology. 2009, 74:1216-1221. 10.1016/j.urology.2009.06.076 